\title{
Article \\ Could Caregivers' Stressful Care Experiences Be Related to Their Attitudes towards Advance Care Planning? A Cross-Sectional Descriptive Study
}

\author{
Pei-Yu Tsai ${ }^{1,2, *}$, Wen-Han Huang ${ }^{2}(\mathbb{D})$ and Yu-Jun Chang ${ }^{3(0)}$ \\ 1 Department of Family Medicine, Department of Hospice-Palliative Care, Changhua Christian Hospital, \\ Changhua 50006, Taiwan \\ 2 Department of Hospice-Palliative Care, Changhua Christian Hospital, Changhua 50006, Taiwan; \\ Hanksky0520@gmail.com \\ 3 Big Data Center, Epidemiology and Biostatistics Center, Changhua Christian Hospital, \\ Changhua 50006, Taiwan; 83686@cch.org.tw \\ * Correspondence: 76934@cch.org.tw; Tel.: +886-4-7238595 (ext. 3267)
}

Citation: Tsai, P.-Y.; Huang, W.-H.; Chang, Y.-J. Could Caregivers' Stressful Care Experiences Be Related to Their Attitudes towards Advance Care Planning? A Cross-Sectional Descriptive Study. Int. J. Environ. Res. Public Health 2021, 18, 9038. https:// doi.org/10.3390/ijerph18179038

Academic Editors: María José Cabañero-Martínez, Manuel Fernández Alcántara and Rafael Montoya Juárez

Received: 1 July 2021

Accepted: 23 August 2021

Published: 27 August 2021

Publisher's Note: MDPI stays neutra with regard to jurisdictional claims in published maps and institutional affiliations.

Copyright: (c) 2021 by the authors. Licensee MDPI, Basel, Switzerland. This article is an open access article distributed under the terms and conditions of the Creative Commons Attribution (CC BY) license (https:// creativecommons.org/licenses/by/ $4.0 /)$.

\begin{abstract}
Previous research has shown that care experiences influence the willingness for advance care planning (ACP). Family caregivers have increased contact with medical providers and procedures in the process of caring, and they have also witnessed the disability and suffering of patients. However, few studies have focused on family caregivers to understand their attitudes towards ACP. The aim of this cross-sectional study was to acknowledge family caregivers' attitudes towards ACP and the related factors, especially care stress and experiences during the care process. We interviewed 291 family caregivers, and the demographics of the caregivers and care recipients, the clinical condition of care recipients, and the caregivers' stress and care experiences were collected via anonymous questionnaires. Multiple logistic regression was performed to determine the factors associated with the attitudes towards ACP. We found that the caregiver having private health insurance $(p<0.001)$ and a completed DNR $(p<0.001)$ and the experience of recipients admitted to the ICU $(p=0.019)$ are associated with caregiver's positive attitudes towards ACP. The greater the stress of conflict within a family over care decisions, the more participants think that ACP is important $(p=0.011)$. It is suggested that (1) in a family-centered culture, a public strategy for promoting ACP could be to emphasize the benefits of ACP in reducing family conflicts, and (2) when people make financial plans, they should also be provided with information about ACP to enable them to form a more integral plan for their future.
\end{abstract}

Keywords: family caregiver; caregiver stress; care experience; advance care planning

\section{Introduction}

Advance care planning (ACP) enables individuals to define goals and preferences for future medical treatment and care, to discuss these goals and preferences with family and healthcare providers, and to record and review these preferences if appropriate [1]. Even in Asian societies that emphasize family participation in an individual's medical decision-making, ACP should be prospective and not be obstructed [2]. In Taiwan, the implementation of ACP is regulated by the "Patient Right to Autonomy Act" [3] which was legislated in late 2015. According to the "Patient Right to Autonomy Act", the declarant, at least one relative of first- or second-degree affinity, and the health care agent shall engage in ACP. After ACP intervention, the declarant writes down the legal advance decision.

Prior studies have shown the factors that influence the individual acceptance of ACP. However, few studies have focused on family caregivers to understand their attitudes towards ACP. Due to caring for disabled relatives, family caregivers have more interactions with medical providers and are exposed to various medical interventions earlier than the 
general public, and they also witness the disability and suffering of care recipients. Do these experiences provoke these caregivers to think about their own values, and make them more willing to discuss their future medical treatment earlier?

Family members may experience various stressful situations during the care process. Stress may be associated with social-cultural variables (employment and education); interpersonal relationships including family responsibilities; and other factors, such as the severity of the recipient's illness, duration of caring and the caregiver's personal health status [4], and the financial burden [5,6]. It remains unclear if, and to what extent, caregivers' stress is related to their attitudes towards ACP.

The purposes of this cross-sectional study were to acknowledge family caregivers' attitudes towards ACP and the related factors, especially care stress and experiences during the care process.

\section{Materials and Methods}

\subsection{Study Design and Setting}

This study was designed as a cross-sectional descriptive study, and recruited family caregivers in central Taiwan, from November 2019 to February 2020. The Institutional Review Board of Changhua Christian Hospital $(\mathrm{CCH})$ approved the study (CCH IRB No. 190909, approval date: 29 October 2019), and all participants signed informed consent. Interviewers were medical staff qualified in nationally recognized ACP consultation training courses or state-certificated core lecturers in the "Patient Right to Autonomy Act". This ensured that each interviewer has a consistent and correct understanding of ACP. Before the formal interview, the research team members reached a consensus about the access process.

The sampling was conducted on general medical, geriatrics, and hospice wards; in home-based settings; in long-term care facilities; and at family-medicine or hospice-care outpatient clinics. To be eligible for inclusion in the study, participants had to have been a caregiver of their adult family members when the care recipients were admitted to wards or at home. The performance status of the care recipients was scored with the Eastern Cooperative Oncology Group (ECOG) performance status scale to assess the level of functioning in their daily living activities [7]. The score ranges from 0 to 5 , indicating from full activity to death. Only the caregivers providing care for recipients with ECOG 2, 3 , or 4 were included in this study, which is to say that the care recipient was restricted in physically strenuous activities. In cases where the care recipient was in a critical condition, their caregiver was excluded from participation.

Before the participant answered the questionnaire, the interviewer used the official ACP literature as an aid to explain ACP to the participant. Then, we assessed the participant's knowledge of ACP with a three-true-false item test. If wrong answers were given, the interviewer immediately clarified the concepts for the participant. These three questions were reviewed by the experts of the ACP team and were found to be reflective of the subject's understanding of ACP.

\subsection{Measures}

The ACP-attitude questionnaire, used to measure a participant's attitude towards $\mathrm{ACP}$, which was adapted from a pilot study [8], consists of four items: "ACP is important," "I will engage in ACP," "I support my family to engage in ACP," and "I agree that ACP can relief family pressure." A self-report 10-point Likert scale was used, where a higher score represents a more positive attitude of the subject. The full score is 40 points. The Cronbach's alpha reliability coefficient for the valid samples collected in this research was $0.89(>0.8)$.

\subsection{Covariates}

Demographic Information, Caregiver Stress, and Care Experiences 
The participants' personal demographic information solicited in this study included their age, gender, marriage status, education attainment, occupation, health status, private health insurance, relationship to care recipient, and completion of a "do not resuscitate" (DNR) document.

The care recipients' personal demographic information solicited included their age, gender, three major diagnoses, completion of a DNR document, and ECOG performance status.

Care experiences, in this article, were defined as the matters that happened during the care process. These care experiences were divided into two groups: (1) (caregivers') care load and (2) (care recipients') suffering. The items in the care load group included the duration of caring, daily caring hours, and whether the caregiver was the medical decision-maker of a care recipient. The items in the suffering group included whether the care recipient had a tube (s) for any purpose, the severity of illness (terminally ill or not), and whether the care recipient had been admitted to the intensive care unit (ICU).

The caregiver's stress was assessed using the Taiwanese version of the Kingston Caregiver Stress Scale (KCSS). The KCSS is primarily a scale to allow family caregivers to express the level of stress that they experience and has been widely used to assess the stress of caregivers of patients with various diseases [9-11]. The KCSS is a 10-item, 5-point self-rating scale where 1 means "no stress" and 5 means "extreme stress". The KCSS includes three domains of stress: caregiving issues, family issues, and financial issues. The scale has good internal consistency and reliability both in the original version [12,13] and in the Taiwanese version (Cronbach's alpha 0.89) [14].

Finally, referring to the method in Hu's study [15], we used a semi-open questionnaire to ask participants why they are willing to engage in ACP (multiple options could be selected).

\subsection{Statistical Analysis}

In order to better understand the relevance of family caregivers' care experiences to caregiving stress and their attitudes towards ACP, we conducted a series of bivariate analyses of caregivers' demographic, psychosocial, and care recipient-related factors affecting caregiver stress and their attitudes towards ACP. The employed statistical tests included Student's $t$-test or one-way ANOVA (for continuous variables) and Chi-square test or Fisher exact test (for categorical variables), as appropriate. In addition, we conducted the Spearman correlation analysis to measure the strength of association between caregiver stress and ACP-attitude. Finally, we employed the multivariable generalized linear regression model controlling possible confounding factors to explore those factors related to ACPattitude. The final model only retained the significant predictors $(p<0.05)$. All data were analyzed using the IBM Corp. Released 2013. IBM SPSS Statistics for Windows, Version 22.0. Armonk, NY: IBM Corp. $p$-Value $<0.05$ was considered statistically significant.

\section{Results}

\subsection{Participant Demographics}

In total, 300 caregivers were approached during the study period, although five caregivers indicated that they were unwilling to be interviewed, thus, the rejection rate was $1.67 \%$. A total of 295 interviews were conducted. Four participants did not complete the questionnaire, meaning a total of 291 valid questionnaires were collected. One of the four participants refused to continue completing the questionnaire due to tiredness, and the three other interviews were interrupted as the participants had to deal with the patients' physiological problems, resulting in a response rate of $98.6 \%$. After the interviewers explained ACP, $93.5 \%$ of the participants on the first attempt correctly answered the three pre-designed questions about knowledge of $\mathrm{ACP}$, which indicated that the participants had a correct understanding of ACP. The mean total score of ACP-attitude was generally high, with 33.4 out of 40 overall ( $\mathrm{SD}=7.9$ ), among which, the four attitude items and the respective mean scores were as follows: "ACP is important," $8.7(\mathrm{SD}=1.8)$; "I will engage 
in $\mathrm{ACP}$, , 7.7 (SD = 2.7); "I support my family to engage in $\mathrm{ACP}$, , $8.3(\mathrm{SD}=2.3)$; and "I agree that ACP can relieve family pressure," 8.7 ( $\mathrm{SD}=2.1$ ), and each score was out of 10 . Overall, the participants of our study were aware of ACP and also showed positive attitudes.

The demographic background of the family caregivers and care recipients in this study are shown in Table 1. The mean ages were 49.9 and 76.9 years for the caregivers and care recipients, respectively. Most of the caregivers were children or children-inlaw $(63.9 \%)$, female $(70.4 \%)$, post-high school educated $(46.7 \%)$, healthy $(69.1 \%)$, in the trade/service industry $(47.1 \%)$, had private health insurance $(84.9 \%)$, and had no completed DNR (88.3\%). Most of the care recipients were female (53.3\%), completely disabled i.e., ECOG performance score $4(45.0 \%)$, and had a completed DNR $(54.3 \%)$. The three major diagnoses were: cardiovascular diseases (44.7\%, including heart failure, hypertension, and coronary artery diseases), cancer $(43.3 \%)$, and organic brain disorder and cerebrovascular diseases (36.1\%).

Table 1. The relationship between the demographics of family caregivers/recipients and KCSS and ACP-attitude.

\begin{tabular}{|c|c|c|c|c|c|c|c|c|c|}
\hline & & \multirow[b]{2}{*}{$n$} & \multirow[b]{2}{*}{$\%$} & \multicolumn{3}{|c|}{ Total KCSS Score } & \multicolumn{3}{|c|}{ Attitude towards ACP } \\
\hline & & & & Mean & SD & $p$-Value & Mean & SD & $p$-Value \\
\hline Total & & 291 & 100.0 & 20.2 & 7.3 & & 33.4 & 7.9 & \\
\hline \multicolumn{10}{|l|}{ Caregivers } \\
\hline \multirow[t]{3}{*}{ Age } & $20-44$ & 110 & 37.8 & 21.0 & 7.1 & 0.061 & 35.0 & 7.2 & 0.013 \\
\hline & $45-64$ & 139 & 47.8 & 20.2 & 7.4 & & 32.8 & 8.0 & \\
\hline & $\geq 65$ & 42 & 14.4 & 17.9 & 7.5 & & 31.1 & 8.9 & \\
\hline \multirow[t]{2}{*}{ Gender } & Male & 86 & 29.6 & 19.8 & 7.0 & 0.555 & 32.5 & 7.9 & 0.210 \\
\hline & Female & 205 & 70.4 & 20.3 & 7.5 & & 33.8 & 7.9 & \\
\hline \multirow[t]{3}{*}{ Health status } & Healthy & 201 & 69.1 & 20.6 & 7.4 & 0.364 & 34.2 & 7.5 & 0.004 \\
\hline & Has chronic disease(s) & 84 & 28.9 & 19.3 & 7.2 & & 32.0 & 8.4 & \\
\hline & Seriously ill & 6 & 2.1 & 19.2 & 6.2 & & 25.5 & 9.0 & \\
\hline \multirow[t]{4}{*}{ Education } & Elementary school & 30 & 10.3 & 18.4 & 7.4 & 0.032 & 30.1 & 9.0 & 0.035 \\
\hline & Junior high school & 42 & 14.4 & 21.2 & 7.3 & & 32.1 & 8.1 & \\
\hline & Senior high school & 83 & 28.5 & 21.8 & 7.8 & & 33.5 & 8.4 & \\
\hline & Post-high school education & 136 & 46.7 & 19.3 & 6.9 & & 34.4 & 7.1 & \\
\hline \multirow[t]{5}{*}{ Occupation } & $\begin{array}{c}\text { Farming fishery and } \\
\text { Pasturage }\end{array}$ & 17 & 5.8 & 18.5 & 4.9 & 0.818 & 30.4 & 9.5 & 0.443 \\
\hline & Public servant & 23 & 7.9 & 20.2 & 7.0 & & 34.6 & 6.9 & \\
\hline & Manufacturing & 47 & 16.2 & 21.0 & 7.1 & & 33.5 & 7.2 & \\
\hline & Trade/service industry & 137 & 47.1 & 20.2 & 7.5 & & 33.8 & 7.7 & \\
\hline & Housekeeper & 67 & 23.0 & 19.9 & 8.0 & & 32.8 & 8.7 & \\
\hline \multirow[t]{2}{*}{ Private health insurance } & Yes & 247 & 84.9 & 20.0 & 7.1 & 0.271 & 34.5 & 7.0 & $<0.001$ \\
\hline & No & 44 & 15.1 & 21.3 & 8.5 & & 27.3 & 9.8 & \\
\hline \multirow[t]{7}{*}{ Relationship to patient } & Spouse & 43 & 14.8 & 21.8 & 8.6 & 0.094 & 33.1 & 7.9 & 0.484 \\
\hline & Child & 129 & 44.3 & 20.6 & 6.9 & & 33.3 & 7.9 & \\
\hline & Grandchild & 34 & 11.7 & 16.6 & 5.4 & & 35.1 & 8.0 & \\
\hline & Child in law & 57 & 19.6 & 19.9 & 7.5 & & 33.8 & 7.8 & \\
\hline & Sibling & 6 & 2.1 & 20.2 & 8.3 & & 28.0 & 9.5 & \\
\hline & Parent & 18 & 6.2 & 20.8 & 7.7 & & 31.6 & 8.2 & \\
\hline & Other & 4 & 1.4 & 20.5 & 9.7 & & 34.5 & 4.1 & \\
\hline \multirow[t]{2}{*}{ Completion of DNR } & Yes & 34 & 11.7 & 20.1 & 7.6 & 0.924 & 38.3 & 3.2 & $<0.001$ \\
\hline & No & 257 & 88.3 & 20.2 & 7.3 & & 32.7 & 8.1 & \\
\hline \multicolumn{10}{|l|}{ Recipients } \\
\hline \multirow[t]{3}{*}{ Age } & $20-44$ & 6 & 2.1 & 22.7 & 10.2 & 0.009 & 30.8 & 8.2 & 0.324 \\
\hline & $45-64$ & 50 & 17.2 & 22.9 & 7.0 & & 34.7 & 6.8 & \\
\hline & $\geq 65$ & 235 & 80.8 & 19.5 & 7.2 & & 33.2 & 8.1 & \\
\hline \multirow[t]{2}{*}{ Gender } & $\bar{M}$ ale & 136 & 46.7 & 20.8 & 7.8 & 0.143 & 34.3 & 6.8 & 0.061 \\
\hline & Female & 155 & 53.3 & 19.6 & 6.9 & & 32.6 & 8.7 & \\
\hline Completion of DNR & Yes & 158 & 54.3 & 21.4 & 7.5 & 0.003 & 33.8 & 7.6 & 0.353 \\
\hline
\end{tabular}


Table 1. Cont.

\begin{tabular}{|c|c|c|c|c|c|c|c|c|c|}
\hline & & \multirow[b]{2}{*}{$n$} & \multirow[b]{2}{*}{$\%$} & \multicolumn{3}{|c|}{ Total KCSS Score } & \multicolumn{3}{|c|}{ Attitude towards ACP } \\
\hline & & & & Mean & SD & $p$-Value & Mean & SD & $p$-Value \\
\hline \multirow{5}{*}{ ECOG } & No & 133 & 45.7 & 18.8 & 6.9 & \multirow{5}{*}{0.010} & 32.9 & 8.3 & \multirow{5}{*}{0.114} \\
\hline & 1 & 55 & 18.9 & 18.0 & 6.7 & & 31.7 & 9.0 & \\
\hline & 2 & 57 & 19.6 & 19.4 & 7.1 & & 34.2 & 6.3 & \\
\hline & 3 & 48 & 16.5 & 22.7 & 7.6 & & 35.2 & 6.5 & \\
\hline & 4 & 131 & 45.0 & 20.5 & 7.4 & & 33.1 & 8.4 & \\
\hline
\end{tabular}

$p$-Value by Student's $t$-test or one-way ANOVA when appropriate. $p$-Value $<0.05$ was considered statistically significant.

\subsection{The ACP-Attitude Relevant to the Demographics of Caregivers Rather Than Recipients}

The demographic backgrounds of the recipients were also related to the caregivers' stress scores. (Table 1) The caregivers reported higher KCSS scores when the recipients were beyond 65 years old $(p=0.009)$, had a poor performance status $(p=0.010)$, or had a completed DNR $(p=0.003)$, but these factors did not affect the caregivers' attitudes towards ACP. By comparison, those caregivers who were younger $(p=0.013)$, had received a highlevel education $(p=0.035)$, had private health insurance $(p<0.001)$, or had a completed DNR $(p<0.001)$ had higher ACP-attitude scores. The caregivers being seriously ill had a negative correlation with their attitudes towards ACP $(p=0.004)$.

We further explored the reasons for the inclination of participants to engage in ACP (Figure 1) and found the top three reasons to be as follows: to avoid the burden on family members in decision making $(70.4 \%)$, to express end-of-life care willingness $(66.7 \%)$, and to avoid conflicts of opinions among family members (60.8\%).

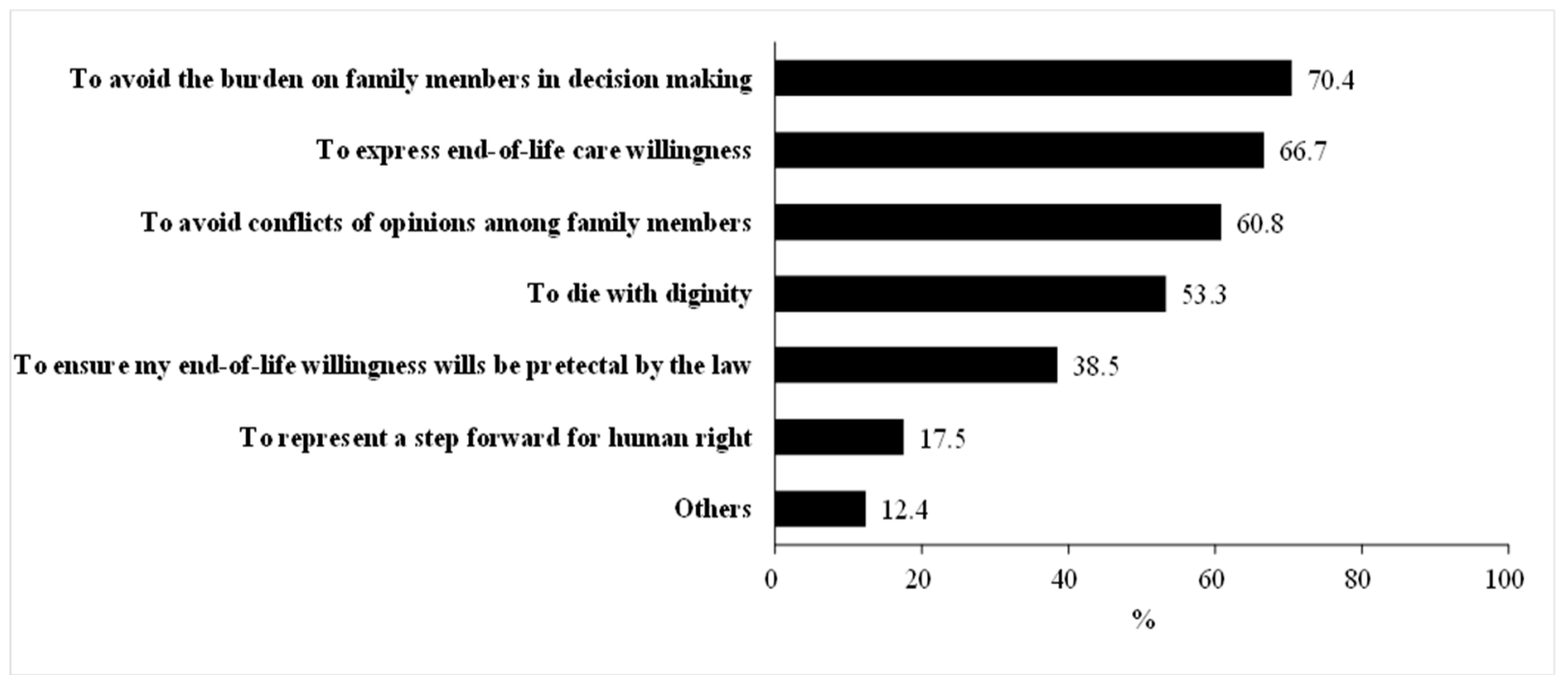

Figure 1. The reasons for willingness to engage in ACP (multiple options could be selected).

\subsection{The Stress Mainly from Family Conflict Correlates with Caregivers Recognition of the Importance of $A C P$}

To further determine the main stress factor relevant to the attitudes towards $\mathrm{ACP}$, we explored the correlation between the various sources of the caregivers' stress and their ACP-attitude (Table 2). The result shows that with a higher level of stress from conflicts among family members over care decisions, the participants were more likely to consider ACP to be important (Spearman's $r=0.150, p=0.011$ ), and they demonstrated a slightly higher ACP-attitude score $(r=0.117, p=0.046)$. 
Table 2. The correlation between the caregiver stress and ACP-attitude.

\begin{tabular}{|c|c|c|c|c|c|c|c|c|c|c|}
\hline \multirow[b]{2}{*}{$(n=291)$} & \multicolumn{2}{|c|}{$\begin{array}{c}\text { ACP } \\
\text { Is Important }\end{array}$} & \multicolumn{2}{|c|}{$\begin{array}{l}\text { Willingness } \\
\text { to Engage }\end{array}$} & \multicolumn{2}{|c|}{$\begin{array}{l}\text { Support My } \\
\text { Family to } \\
\text { Engage }\end{array}$} & \multicolumn{2}{|c|}{$\begin{array}{c}\text { Reduce } \\
\text { Family's Stress }\end{array}$} & \multicolumn{2}{|c|}{$\begin{array}{c}\text { Attitude } \\
\text { towards ACP }\end{array}$} \\
\hline & $r$ & $p$-Value & $r$ & $p$-Value & $r$ & $p$-Value & $r$ & $p$-Value & $r$ & $p$-Value \\
\hline Care giving issues & 0.059 & 0.314 & 0.008 & 0.885 & 0.050 & 0.393 & 0.048 & 0.414 & 0.058 & 0.322 \\
\hline Over-burdened & 0.001 & 0.989 & 0.030 & 0.613 & 0.006 & 0.915 & 0.000 & 0.998 & 0.000 & 0.999 \\
\hline $\begin{array}{l}\text { Change in your } \\
\text { relationship }\end{array}$ & 0.088 & 0.133 & 0.027 & 0.642 & 0.031 & 0.593 & 0.062 & 0.295 & 0.070 & 0.233 \\
\hline Changes in your social life & -0.028 & 0.630 & -0.028 & 0.636 & 0.007 & 0.910 & -0.024 & 0.682 & -0.008 & 0.896 \\
\hline $\begin{array}{l}\text { Conflicts with your } \\
\text { previous daily } \\
\text { commitments }\end{array}$ & -0.018 & 0.760 & -0.022 & 0.706 & 0.006 & 0.921 & 0.020 & 0.734 & 0.015 & 0.793 \\
\hline $\begin{array}{l}\text { Trapped by the } \\
\text { responsibilities }\end{array}$ & 0.064 & 0.276 & 0.013 & 0.827 & 0.048 & 0.413 & 0.040 & 0.493 & 0.061 & 0.298 \\
\hline $\begin{array}{c}\text { A lack of confidence in } \\
\text { your ability }\end{array}$ & 0.040 & 0.493 & -0.036 & 0.543 & 0.029 & 0.627 & 0.064 & 0.276 & 0.033 & 0.574 \\
\hline $\begin{array}{l}\text { Concerns regarding the } \\
\text { future care needs }\end{array}$ & 0.120 & 0.041 & 0.025 & 0.677 & 0.104 & 0.077 & 0.116 & 0.048 & 0.108 & 0.065 \\
\hline Family issues & 0.124 & 0.035 & 0.057 & 0.332 & 0.036 & 0.538 & 0.053 & 0.363 & 0.099 & 0.091 \\
\hline $\begin{array}{l}\text { Conflicts within your } \\
\text { family over care decisions }\end{array}$ & 0.150 & 0.011 & 0.064 & 0.277 & 0.059 & 0.320 & 0.081 & 0.170 & 0.117 & 0.046 \\
\hline $\begin{array}{c}\text { Conflicts over the amount } \\
\text { of support }\end{array}$ & 0.071 & 0.229 & 0.057 & 0.337 & 0.014 & 0.814 & 0.028 & 0.631 & 0.073 & 0.215 \\
\hline Financial difficulties & 0.044 & 0.457 & 0.082 & 0.161 & 0.053 & 0.369 & 0.095 & 0.105 & 0.078 & 0.186 \\
\hline Financial issues & 0.044 & 0.457 & 0.082 & 0.161 & 0.053 & 0.369 & 0.095 & 0.105 & 0.078 & 0.186 \\
\hline Total KCSS score & 0.077 & 0.188 & 0.019 & 0.751 & 0.056 & 0.345 & 0.058 & 0.323 & 0.080 & 0.173 \\
\hline
\end{tabular}

$r$ : Spearman's correlation coefficient.

Additionally, the greater the stress about "concerns regarding the future care needs," the more the participants thought that ACP is important $(r=0.120, p=0.041)$, and they agreed slightly more that ACP can reduce the stress of family members $(r=0.116$, $p=0.048)$. However, we found no significant correlation of the KCSS score with ACPattitude $(p=0.173)$.

3.4. Various Care Experiences Cause Different Aspects of Stress, While Only the Care Recipients' Former ICU-Admission Is Associated with the Caregivers'Positive ACP-Attitude

The relationship between caregivers' care experiences and stress and their attitudes towards ACP are shown in Table 3. In the caregivers' care load group, when the daily caring hours were more than $8 \mathrm{~h}$, the KCSS scores were higher $(p=0.001)$, especially regarding stresses in the caregiving issue domain $(p<0.001)$ and in the financial issue domain $(p=0.011)$. Being a care recipient's medical decision-maker also brought about higher scores in KCSS $(p<0.001)$, particularly attributed to both the caregiving $(p<0.001)$ and financial issues $(p=0.011)$. However, the duration of caring did not show a statistically significant difference in any of the caregivers' stress domains.

In the recipient suffering group, when a care recipient was terminally ill, the caregivers reported higher scores in the caregiving issue domain, the family issues domain, and the total KCSS score $(p<0.001, p=0.010$, and $p<0.001$, respectively). In instances where a recipient had been admitted to the ICU, the stress was higher in the caregiving issues domain $(p=0.001)$ and also in the total KCSS score $(p=0.003)$. In cases where a recipient had a tube(s), the stress was higher in the caregiving issues domain $(p=0.019)$ and in the total KCSS score $(p=0.021)$. In summary, every aspect of care experiences, except the duration of caring, puts caregivers under pressure from different domains. However, among the various stressful care experiences, only the experience of providing care for recipients who had been admitted to the ICU significantly increased the caregivers' ACP-attitude score. $(p=0.019)$. 
Table 3. The relationship between the caregivers' care experience and stress and their attitude towards ACP.

\begin{tabular}{|c|c|c|c|c|c|c|c|c|c|c|c|c|c|c|c|c|c|}
\hline \multirow[b]{2}{*}{ Care Experience } & & \multirow[b]{2}{*}{$n$} & \multicolumn{3}{|c|}{ Care Giving Issues * } & \multicolumn{3}{|c|}{ Family Issues ** } & \multicolumn{3}{|c|}{ Financial Issues *** } & \multicolumn{3}{|c|}{ Total KCSS Score } & \multicolumn{3}{|c|}{ Attitude towards ACP } \\
\hline & & & Mean & SD & $p$-Value & Mean & SD & $p$-Value & Mean & SD & $p$-Value & Mean & SD & $p$-Value & Mean & SD & $p$-Value \\
\hline Total & & 291 & 14.6 & 5.6 & & 3.8 & 1.9 & & 1.7 & 1.1 & & 20.2 & 7.3 & & 33.4 & 7.9 & \\
\hline \multicolumn{18}{|l|}{ Caregivers' care load } \\
\hline \multirow[t]{3}{*}{ Duration of caring } & $<1$ year & 103 & 15.1 & 6.1 & 0.211 & 3.7 & 1.9 & 0.260 & 1.8 & 1.1 & 0.057 & 20.6 & 7.9 & 0.139 & 34.2 & 7.7 & 0.117 \\
\hline & $1-3$ years & 50 & 15.4 & 5.7 & & 4.2 & 2.1 & & 2.0 & 1.1 & & 21.6 & 7.8 & & 34.6 & 7.5 & \\
\hline & $>3$ years & 138 & 14.0 & 5.2 & & 3.7 & 1.9 & & 1.6 & 0.9 & & 19.3 & 6.7 & & 32.4 & 8.2 & \\
\hline \multirow[t]{3}{*}{ Daily caring hours } & $<8 \mathrm{~h}$ & 149 & 13.2 & 5.0 & $<0.001$ & 3.8 & 1.9 & 0.249 & 1.6 & 0.9 & 0.011 & 18.6 & 6.7 & 0.001 & 33.3 & 7.9 & 0.824 \\
\hline & $8-16 \mathrm{~h}$ & 52 & 16.2 & 6.2 & & 4.1 & 1.9 & & 1.9 & 1.2 & & 22.2 & 8.0 & & 34.0 & 7.0 & \\
\hline & $>16 \mathrm{~h}$ & 90 & 16.0 & 5.7 & & 3.6 & 1.9 & & 2.0 & 1.1 & & 21.5 & 7.5 & & 33.1 & 8.5 & \\
\hline \multirow{3}{*}{$\begin{array}{c}\text { Patient's medical } \\
\text { decision maker } \\
\text { Recipients' suffering }\end{array}$} & Yes & 161 & 15.9 & 5.5 & $<0.001$ & 4.0 & 2.1 & 0.073 & 1.9 & 1.1 & 0.001 & 21.8 & 7.2 & $<0.001$ & 33.1 & 8.0 & 0.568 \\
\hline & No & 130 & 13.1 & 5.4 & & 3.6 & 1.8 & & 1.5 & 0.9 & & 18.2 & 7.1 & & 33.7 & 7.8 & \\
\hline & & & & & & & & & & & & & & & & & \\
\hline \multirow[t]{2}{*}{ Terminally ill } & Yes & 146 & 15.9 & 5.7 & $<0.001$ & 4.1 & 2.1 & 0.010 & 1.8 & 1.1 & 0.103 & 21.8 & 7.5 & $<0.001$ & 34.0 & 7.1 & 0.192 \\
\hline & No & 145 & 13.4 & 5.3 & & 3.5 & 1.7 & & 1.6 & 1.0 & & 18.5 & 6.8 & & 32.8 & 8.7 & \\
\hline \multirow{5}{*}{$\begin{array}{c}\text { Has been admitted } \\
\text { to ICU } \\
\text { Has tube(s) for } \\
\text { any purpose }\end{array}$} & Yes & 133 & 15.8 & 5.7 & 0.001 & 4.0 & 2.1 & 0.058 & 1.8 & 1.2 & 0.727 & 21.6 & 7.4 & 0.003 & 34.6 & 7.6 & 0.019 \\
\hline & No & 158 & 13.7 & 5.4 & & 3.6 & 1.8 & & 1.7 & 0.9 & & 19.0 & 7.1 & & 32.4 & 8.1 & \\
\hline & 0 & 135 & 13.6 & 5.5 & 0.019 & 3.6 & 1.7 & 0.272 & 1.7 & 1.0 & 0.524 & 18.9 & 7.1 & 0.021 & 32.9 & 7.9 & 0.353 \\
\hline & 1 & 86 & 14.9 & 5.6 & & 3.8 & 2.0 & & 1.7 & 1.0 & & 20.5 & 7.3 & & 33.2 & 7.8 & \\
\hline & $\geq 3$ & 13 & 16.4 & 7.3 & & 4.2 & 1.7 & & 2.1 & 1.6 & & 22.7 & 8.9 & & 32.4 & 10.5 & \\
\hline
\end{tabular}

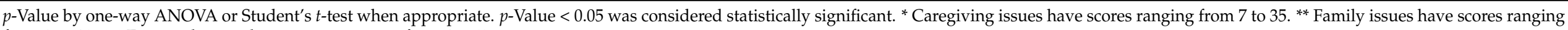
from 2 to 10 . ${ }^{* *}$ Financial issues have scores ranging from 1 to 5 . 
Controlling for possible confounding factors in the multivariable generalized linear model, a positive ACP-attitude was associated with caregivers with the following three conditions: seriously ill ( $95 \% \mathrm{CI}:-14.589-3.002 ; p=0.003)$, with private health insurance (95\% CI: 4.640-9.442; $p<0.001$ ), and with a completed DNR (95\% CI: 2.292-7.424; $p<0.001$ ). ACP-attitude was also positively related to the care experience of caring for recipients who had been admitted to ICU (95\% CI: $0.214-3.523 ; p=0.027$ ) (Table 4$)$.

Table 4. The results of generalized linear models on the attitude towards ACP.

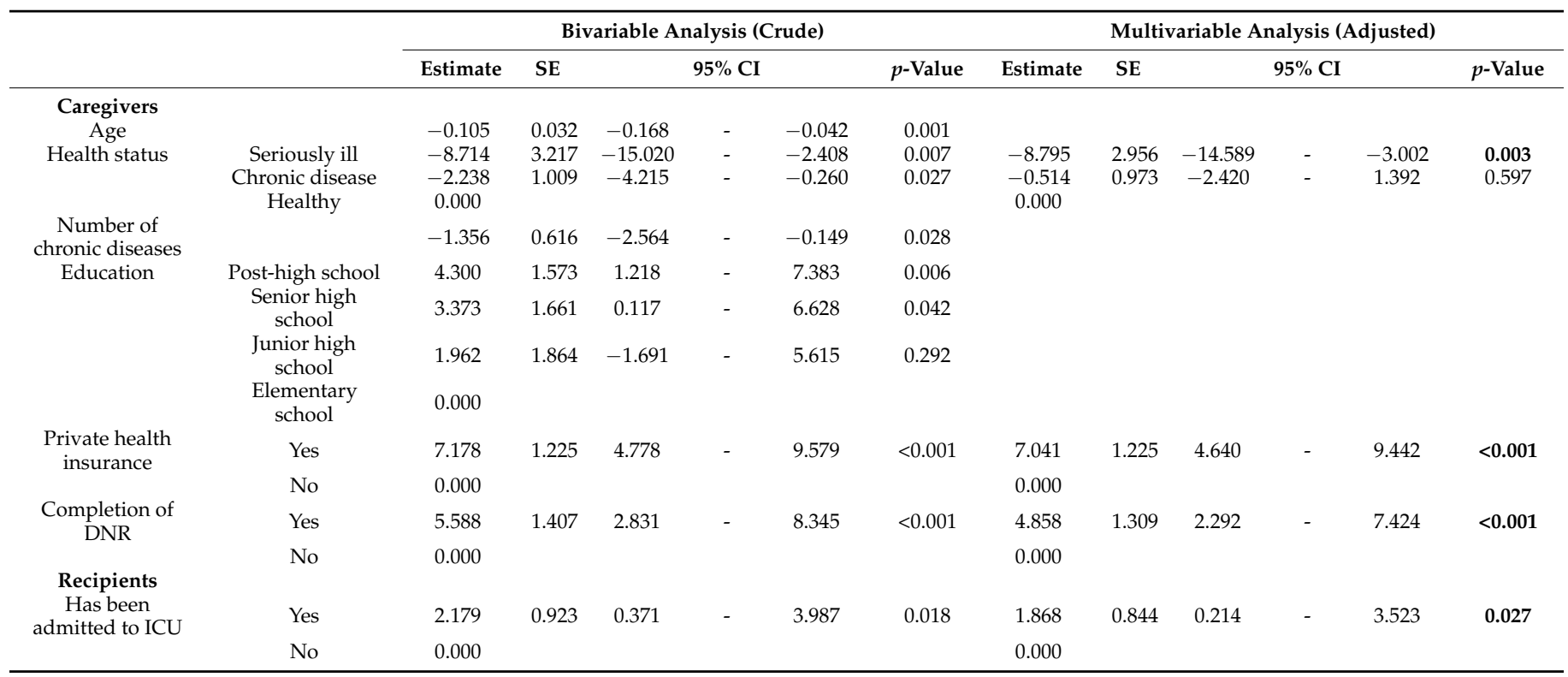

\section{Discussion}

This study aimed to understand family caregivers' attitudes towards ACP and the related factors. The ACP-attitude is relevant to the demographics of caregivers rather than recipients. Caregivers' stress mainly from family conflicts, correlates with their recognition of the importance of ACP. Various care experiences cause different aspects of stress, while only care recipients' ICU -admissions are associated with caregivers' positive ACP-attitude.

The results of our study indicated that the caregiver's health status, private health insurance, and a completed formal DNR document are the main factors that affect their attitudes towards ACP. In our study, seriously ill caregivers showed more negative attitudes towards ACP than those who were healthy or had a chronic disease. The reason for this might be that the issues discussed in ACP about terminal illness, or fatal conditions, which may soon be confronted by seriously ill caregivers, could impose unpleasant feelings [16], leading to a negative attitude towards ACP. However, with the small sample size of the subgroup $(n=6)$ and the high variation in the ACP-attitude score, more samples are needed for further research.

Our study revealed that caregivers with private health insurance had a higher ACPattitude score, which echoes the findings of a previous study in Australia, i.e., that undertaking a wider future planning process (e.g., making a will or financial enduring power of attorney) is a trigger for engaging ACP [17]. A reasonable explanation for this is that an insured person may have the trait or habit of planning ahead for important life occurrences, and is, therefore, more willing to plan for finance and health care issues in advance. Similarly, those who had a completed formal DNR had already put their future medical preferences into perspective and taken action to actualize them in the form of a document. Therefore, it is reasonable that DNR signees hold more positive attitudes towards ACP.

Similar to other studies [14,18], our research also found that the stress of family conflict is positively correlated with attitudes towards ACP; however, the correlation was weak. Analyzing the relationship between KCSS score and ACP-attitude, we observed that those 
caregivers who had experienced greater stress from conflicts within family members over medical decisions, assigned more importance to ACP. From the answers to the semi-open questionnaire, the main reasons for a caregivers' willingness to engage in ACP were related to family concerns, such as: sparing loved ones the suffering of making difficult decisions and avoiding conflict of opinions. This finding adds another layer to the consensus of the current literature, proposing that the motivation for engaging in ACP is to avoid a burden on the family $[19,20]$. ACP is a process that encourages individuals and their families to express one another's thoughts and emotions. Through communication and negotiation among patients and family members, a consensus can be reached, which can resolve possible family conflicts in the future $[16,21]$. Those caregivers who had experienced family conflicts during the care process were more likely to try to avoid exerting the same pressure on their family in the future, and would therefore consider the option of ACP and make care decisions for themselves in advance. Taiwan, similar to most other Asian countries, has a family-centered culture. Family-centered ethics posit that end-of-life care decisions are an intra-family matter, resulting in family members adopting uncertain attitudes towards ACP [21]. Most people are reluctant to admit conflicts in their families. A Chinese proverb states that a "harmonious family can lead to the success of everything." Taiwanese people are worried that hidden intra-family conflict may come to the fore during the ACP process. Although they think ACP is important, they hesitate to engage in ACP (Table 3), and their attitudes towards ACP are relatively weakly positive. Understanding the cultural context of family conflict and acknowledging this issue during the ACP process can guide the declarant in improving communication quality.

Previous studies carried out in different cultural contexts have shown that witnessing the suffering of relatives and friends influences the willingness of caregivers to engage in ACP $[8,18-20,22,23]$. Chiang et al. [20] also pointed out that compared to the general public, family caregivers who provide care for disabled or seriously ill relatives are more positive and willing to accept the ACP concept. Our study further revealed that not all experiences are related to ACP-attitude. The results showed that care load experience has no correlation with ACP-attitude. In contrast, the experience of witnessing suffering is positively correlated with the attitudes towards $A C P$, specifically the suffering from ICU admission of care recipients, but not from recipients' intubation (Table 3). In Chinesebased culture, reduced food intake or the inability to eat via the mouth are considered problematic. Most patients or family members would then choose artificial nutrition and hydration. According to data from the Ministry of Health and Welfare of Taiwan, up to 9.6\% of hospitalized patients had a nasogastric tube inserted for enteral nutrition in 2020 [24]. A study reported that $29.2 \%$ of elderly people at long-term care facilities in Taipei, Taiwan, were fed through a tube [25]. This suggests that intubation for medical care is common and generally acceptable among Taiwanese people and explains why the presence of tubing is not related to the attitudes of caregivers towards ACP. In contrast, recipients' suffering in the ICU has a greater positive impact on caregivers' ACP-attitude. The exact reason for this needs further study.

Certain care experiences were associated with a higher KCSS score, especially in the caregiving issue domain. The study by Mok et al. found that Chinese people in Hong Kong, influenced by their culture and religion, accept the responsibility for taking care of an ill family member as a normal course of life or fate [26]. In Asia, many countries and regions are strongly influenced by Confucianism, Buddhism, and Taoism, such as China, Japan, Korea, Singapore, Hong Kong, and Taiwan. They share similar family ethics and filial piety. A common belief is that people who submit to fate will lead to a good life [27]. However, as found in this study, either from the care load or from witnessing the suffering of recipients, caregivers are under care pressure. Obviously, the statement that "caring for your family is your responsibility and fate" does not ease the pressure in the care process.

Financial factors might be a consideration in medical care decisions [28]. Financial stress is perceived once caregivers are also the medical decision-makers, especially when considering all of the consequences of medical care decisions, including the substantial 
burdens of logistic, finance, and care loads that may arise. For example, after treatment, patients could be disabled and bed-ridden for a long time, which often exacts a significant financial burden on their families.

When a patient is in the terminal stage of life, the caregiver's family-issue stress increases. During this period, family members are often called upon to make end-of-life decisions, including whether to initiate, withhold, continue, or withdraw life-sustaining treatment. Families sometimes come into conflict, as multiple family members participate in the decision-making process [29], especially when they do not know the recipients wishes $[30,31]$.

We acknowledge limitations to our study. First, we may need to take cultural context into account to properly interpret the results of this study. One of our findings is that compared to financial and caregiving issues, the ACP-attitude has a stronger association with the family-issue stress domain, which may not be analogous to other different cultures due to the intrinsic family-centered propensity of Taiwanese people. Second, we noticed that most of the participants showed a positive attitude towards ACP, possibly due to the "socially acceptable" response, rather than their true view of ACP [32]. This could have increased the ACP-attitude scores in general, thereby making it difficult for us to observe the differences in correlations between variates. Additionally, limited by the inherent disadvantage of a personal interview survey, one must be extremely cautious when interpreting attitudes towards ACP.

\section{Conclusions}

The study provided insights into family caregivers' attitudes towards ACP and related factors. First, people with health insurance have more positive attitudes towards ACP. This result reminds us that when people undertake financial planning, they should also be provided with information about ACP to enable them to form a more integral plan for their future. Second, the results showed that care experiences, including the (caregivers') care load and (recipients') suffering, cause care pressure. However, no relationship was observed between the care load and ACP-attitude. On the contrary, ACP-attitude was positively related to the caregivers' witnessing the patients' suffering in the ICU. Therefore, for those whose family members have been admitted to ICU, we can provide them with information about $\mathrm{ACP}$ in a timely manner and encourage them to engage in ACP. When an ACP declarant mentions the experience of taking care of others as the motivation for engaging in ACP, it is necessary for the ACP team to further clarify the kind of stressful experience that has had that impact in order to conduct more efficient dialogues and to provide the full benefits of ACP. Third, caregivers facing stress from intra-family conflict acknowledge the importance of ACP more. This could be further supported by our finding that caregivers are significantly motivated to engage in $\mathrm{ACP}$ due to the expectations that ACP could help to relieve family suffering as a result of decision making and to avoid conflicts of opinions. In the clinic, we need to provide more support to medical decisionmakers. Providing information about ACP to families with terminally ill relatives could be especially important in assisting them in making end-of-life medical decisions, as it was shown that these families experience the most stress from internal issues. In a familycentered culture, the benefits of ACP in reducing family conflict can be emphasized as a strategy for promoting ACP.

Author Contributions: P.-Y.T. conceived the study and had oversight of the research, including study design, ethical approval, and invitation of research participants; P.-Y.T. also conducted interviews with participants and led writing and revision of the manuscript; Y.-J.C. and W.-H.H. made substantial contributions to the analysis of data and advised in the preparation of the manuscript; W.-H.H. also participated in writing and conducted interviews with participants. All authors have read and agreed to the published version of the manuscript.

Funding: This research was supported by 2020 Changhua Christian Hospital Research Project Funding under award number 037. 
Institutional Review Board Statement: The Institutional Review Board of Changhua Christian Hospital (CCH) approved the study (CCH IRB No. 190909, approval date: 29 October 2019).

Informed Consent Statement: All participants signed informed consent.

Data Availability Statement: The datasets generated and analyzed during the current study are not publicly available due to maintenance of confidentiality and privacy requirements consistent with the Institutional Review Board approval.

Acknowledgments: The authors are thankful to all the participants who are graciously shared their time and experiences with us. Thanks to Robert W. Hopkins for permitting us to use KCSS Taiwanese version.

Conflicts of Interest: The authors declare no conflict of interest. The funding source of this study had no role in the design of this study and will not have any role during its execution, analyses, interpretation of the data, or decision to submit results.

\section{References}

1. Rietjens, J.A.C.; Sudore, R.L.; Connolly, M.; van Delden, J.J.; Drickamer, M.A.; Droger, M.; van der Heide, A.; Heyland, D.K.; Houttekier, D.; Janssen, D.J.A.; et al. Definition and recommendations for advance care planning: An international consensus supported by the European Association for Palliative Care. Lancet Oncol. 2017, 18, e543-e551. [CrossRef]

2. $\quad$ Lin, C.P.; Cheng, S.Y.; Mori, M.; Suh, S.Y.; Chan, H.Y.; Martina, D.; Pang, W.S.; Huang, H.L.; Peng, J.K.; Yao, C.A.; et al. 2019 Taipei Declaration on Advance Care Planning: A Cultural Adaptation of End-of-Life Care Discussion. J. Palliat. Med. 2019, 22, 1175-1177. [CrossRef] [PubMed]

3. Patient Right to Autonomy Act. Available online: https://law.moj.gov.tw/ENG/LawClass/LawAll.aspx?pcode=L0020189 (accessed on 29 April 2020).

4. Chiao, C.Y.; Wu, H.S.; Hsiao, C.Y. Caregiver burden for informal caregivers of patients with dementia: A systematic review. Int. Nurs. Rev. 2015, 62, 340-350. [CrossRef]

5. Duggleby, W.; Tycholiz, J.; Holtslander, L.; Hudson, P.; Nekolaichuk, C.; Mirhosseini, M.; Parmar, J.; Chambers, T.; Alook, A.; Swindle, J. A metasynthesis study of family caregivers' transition experiences caring for community-dwelling persons with advanced cancer at the end of life. Palliat. Med. 2017, 31, 602-616. [CrossRef]

6. Hawkley, L.C.; Capitanio, J.P. Perceived social isolation, evolutionary fitness and health outcomes: A lifespan approach. Philos. Trans. R. Soc. Lond. B Biol. Sci. 2015, 370, 20140114. [CrossRef]

7. ECOG Performance Status. Available online: https://ecog-acrin.org/resources/ecog-performance-status (accessed on 1 May 2021).

8. Tsai, P.Y.; Hsieh, Y.C.; Chung, M.C.; Hsu, J.M. The Practice and Prospect of Advance Care Planning Outpatient Clinic. Taizwan J. Hosp. Palliat. Care 2019, 23, 155-167. [CrossRef]

9. Petrikis, P.; Baldouma, A.; Katsanos, A.H.; Konitsiotis, S.; Giannopoulos, S. Quality of Life and Emotional Strain in Caregivers of Patients with Multiple Sclerosis. J. Clin. Neurol. 2019, 15, 77-83. [CrossRef]

10. Bhattacharjee, A.; Banerjee, A. Impact of Caregiver's Stress on Cancer Patient's Level of Anxiety and Depression. Indian J. Health Wellbeing 2018, 9, 530-533.

11. Ramachandrana, A.; Vyasb, N.; Pothiyilc, D.I. Stress among the caregivers of mentally disabled children visiting a rehabilitation centre in Chennai, Tamil Nadu-A cross-sectional study. Clin. Epidemiol. Glob. Health 2020, in press. [CrossRef]

12. Hopkins, R.W.; Kilik, L.A. The Kingston Caregiver Stress Scale (KCSS). Available online: http://nebula.wsimg.com/979c665ffd4 1f5c5a0f8987c3817d273? AccessKeyId=954A289F7CDF75707C10\&disposition=0\&alloworigin=1 (accessed on 20 April 2020).

13. Sadak, T.; Korpak, A.; Wright, J.D.; Lee, M.K.; Noel, M.; Buckwalter, K.; Borson, S. Psychometric Evaluation of Kingston Caregiver Stress Scale. Clin. Gerontol. 2017, 40, 268-280. [CrossRef]

14. Tseng, H.F. Family Caregiver's Stress, Burden and Well-Being among Patient with Dementia; National Taipei University of Nursing and Health Sciences: Taipei, Taiwan, 2014.

15. Lin, H.M.; Yang, C.L.; Chen, M.M.; Chiu, T.Y.; Hu, W.Y. Inpatients' Willingness on and Acceptance of Promotion for Signing of Advance Directives. Taiwan J. Hosp. Palliat. Care 2011, 16, 281-295.

16. Zwakman, M.; Jabbarian, L.J.; van Delden, J.; van der Heide, A.; Korfage, I.J.; Pollock, K.; Rietjens, J.; Seymour, J.; Kars, M.C. Advance care planning: A systematic review about experiences of patients with a life-threatening or life-limiting illness. Palliat. Med. 2018, 32, 1305-1321. [CrossRef]

17. White, B.; Tilse, C.; Wilson, J.; Rosenman, L.; Strub, T.; Feeney, R.; Silvester, W. Prevalence and predictors of advance directives in Australia. Intern. Med. J. 2014, 44, 975-980. [CrossRef]

18. Amjad, H.; Towle, V.; Fried, T. Association of experience with illness and end-of-life care with advance care planning in older adults. J. Am. Geriatr. Soc. 2014, 62, 1304-1309. [CrossRef]

19. Levi, B.H.; Dellasega, C.; Whitehead, M.; Green, M.J. What influences individuals to engage in advance care planning? Am. J. Hosp. Palliat. Care 2010, 27, 306-312. [CrossRef] 
20. Chiang, F.M.; Hsieh, J.G.; Fan, S.Y.; Wang, Y.W.; Wang, S.C. Does the Experience of Caring for a Severely Disabled Relative Impact Advance Care Planning? A Qualitative Study of Caregivers of Disabled Patients. Int. J. Environ. Res. Public Health $2020,17$. [CrossRef]

21. Hong, M.; Yi, E.H.; Johnson, K.J.; Adamek, M.E. Facilitators and Barriers for Advance Care Planning Among Ethnic and Racial Minorities in the U.S.: A Systematic Review of the Current Literature. J. Immigr. Minor. Health 2018, 20, 1277-1287. [CrossRef]

22. Carr, D. "I don't want to die like that ... ": The impact of significant others' death quality on advance care planning. Gerontologist 2012, 52, 770-781. [CrossRef]

23. van Wijmen, M.P.; Pasman, H.R.; Widdershoven, G.A.; Onwuteaka-Philipsen, B.D. Motivations, aims and communication around advance directives: A mixed-methods study into the perspective of their owners and the influence of a current illness. Patient. Educ. Couns. 2014, 95, 393-399. [CrossRef]

24. Nasogastric tube Feeding Prevalence in Taiwan. Available online: https://dep.mohw.gov.tw/dos/cp-1720-7414-113.html (accessed on 20 May 2021).

25. Lin, L.C.; Wu, S.C.; Chen, H.S.; Wang, T.G.; Chen, M.Y. Prevalence of impaired swallowing in institutionalized older people in taiwan. J. Am. Geriatr. Soc. 2002, 50, 1118-1123. [CrossRef] [PubMed]

26. Mok, E.; Lai, C.K.; Wong, F.L.; Wan, P. Living with early-stage dementia: The perspective of older Chinese people. J. Adv. Nurs. 2007, 59, 591-600. [CrossRef]

27. Chan, S.W. Family caregiving in dementia: The Asian perspective of a global problem. Dement. Geriatr. Cogn. Disord. 2010, 30, 469-478. [CrossRef]

28. McNeil, B.J.; Keller, E.; Adelstein, S.J. Primer on certain elements of medical decision making. N. Engl. J. Med. 1975, 293, 211-215. [CrossRef]

29. Trees, A.R.; Ohs, J.E.; Murray, M.C. Family Communication about End-of-Life Decisions and the Enactment of the Decision-Maker Role. Behav. Sci. 2017, 7, 36. [CrossRef] [PubMed]

30. Bollig, G.; Gjengedal, E.; Rosland, J.H. They know!-Do they? A qualitative study of residents and relatives views on advance care planning, end-of-life care, and decision-making in nursing homes. Palliat. Med. 2016, 30, 456-470. [CrossRef] [PubMed]

31. Kramer, B.J.; Boelk, A.Z.; Auer, C. Family conflict at the end of life: Lessons learned in a model program for vulnerable older adults. J. Palliat. Med. 2006, 9, 791-801. [CrossRef] [PubMed]

32. Menon, S.; Kars, M.C.; Malhotra, C.; Campbell, A.V.; van Delden, J.J.M. Advance Care Planning in a Multicultural Family Centric Community: A Qualitative Study of Health Care Professionals', Patients', and Caregivers' Perspectives. J. Pain Symptom. Manag. 2018, 56, 213-221. [CrossRef] 